Archive for

Organic Chemistry

Arkivoc 2018, part vii, 50-57

\title{
An alternative stereoselective total synthesis of Verbalactone
}

\author{
Sreelatha Vanjivaka, ${ }^{a}$ Kakarla Ramanakumar, ${ }^{b}$ M. Rajeswari, ${ }^{c}$ Jyothi Vantikommu, ${ }^{a}$ Gattu Sridhar, ${ }^{d}$ \\ and Sadanandam Palle ${ }^{a^{*}}$ \\ ${ }^{a}$ Centre for Chemical Sciences \& Technology, Institute of Science \& Technology, Jawaharlal Nehru \\ Technological University, Hyderabad - 500 085, India \\ ${ }^{b}$ Department of Chemistry, CMR Institute of Technology, Medchal, Secunderabad - 01, India \\ ${ }^{c}$ Organic Chemistry Department, Telangana university, Dichpally, Nizamabad, Telangana, India \\ ${ }^{d}$ Organic and Biomolecular Chemistry Division, CSIR-Indian Institute of Chemical Technology, Hyderabad, \\ 500107, India \\ E-mail: rk1org@yahoo.com
}

Received 02-11-2018

Accepted 05-31-2018

Published on line 08-28-2018

\section{Abstract}

A simple and efficient synthesis of Verbalactone has been accomplished from inexpensive and commercially available starting material, hexanal. This concise synthesis utilizes stereoselective reduction of $\beta$-hydroxyketone using catecholborane, regioselective opening of epoxide and Yamaguchi reaction for the construction of the macrolactone.

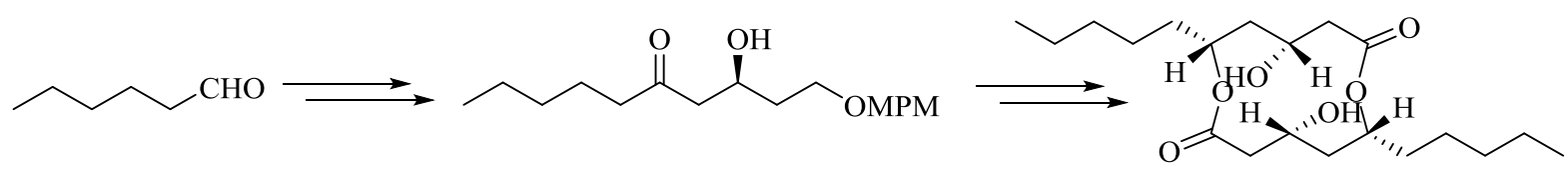

Keywords: Hexanal, regioselective epoxide opening, Yamaguchi macrolactonisation, stereoselective synthesis, Verbalactone 


\section{Introduction}

Macrolides are large lactone ring molecules and these molecules are derived by the internal esterification of the corresponding hydoxy acids. Macrodiolides and macrocyclic monolactones are two types of macrolides. Researchers across the world concentrated towards the synthesis of both homo and hetero dimers (macro dilactones) $)^{1-3}$ and macrocyclic monolactones ${ }^{4-6}$. Macrodiolides are interesting natural products which can be isolated from marine sponges and different fungi. These macrodiolides exhibited potent biological activities including antihelmintic, ${ }^{7,8}$ antifungal ${ }^{9,10}$ and phytotoxic activities ${ }^{11}$.

Verbalactone 1 is a 12 membered $C_{2}$ symmetric dilactone and it was isolated from the roots of Verbascum undulatum Lam., a biennial plant of the genus Verbascum that belongs to the family Scrophulariacea by Mitaku et al. ${ }^{12}$ It showed antibacterial activity against Gram-positive (MIC $=62.5 \mathrm{mg} / \mathrm{cm}^{3}$ ) and Gram-negative bacteria $\left(\mathrm{MIC}=125 \mathrm{mg} / \mathrm{cm}^{3}\right.$ ). Verbalactone $(\mathbf{1})$ is thus a dimeric lactone with $\mathrm{C}_{2}$-symmetry and has a NMR profile similar to the monomer lactone of $(3 R, 5 R)$-dihydroxydecanoic acid. ${ }^{13-16}$ The structure and absolute stereochemistry of verbalactone was detemined as $4 R, 6 R, 10 R, 12 R$ by the $1 \mathrm{D}$ and $2 \mathrm{D}$ NMR spectroscopic methods and chemical correlations. The NMR profile of 1 is similar to the NMR data of $(3 R, 5 R)$ dihydroxydecanoic acid. ${ }^{13-16}$ The structure of verbalactone was shown in Figure 1.

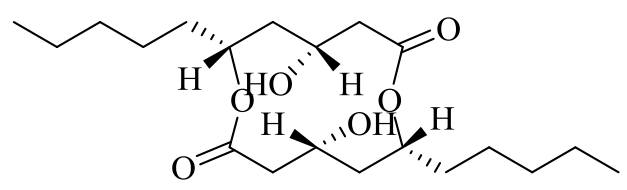

Verbalactone (1)

\section{Figure 1}

Meanwhile, several scientists reported the total synthesis of Verbalactone due to its interesting biologically active nature and stereochemical complexicity. ${ }^{17-27}$ The reported methods involve long reaction sequences, low yields and dependence of chiral pool resources were disadvantages. In continuation of our research work on the synthesis of biologically active natural products, herein we report an efficient straightforward and an alternative concise synthetic route for stereoselective total synthesis of Verbalactone starting from commercially available material hexanal with overall high yield.

\section{Results and Discussion}

Our retrosynthetic analysis of $\mathbf{1}$ is outlined in Scheme 1. Retrosynthetically (Scheme 1), the target molecule 1 could be obtained from dihydroxy acid $\mathbf{2}$ by Yamaguchi's macrolactonization. While compound $\mathbf{2}$ could be obtained from opening of epoxide 4 with 2-pentyl-1,3-dithiane 3, the 1,3-dithiane $\mathbf{3}$ could be prepared from the aldehyde 5 . 


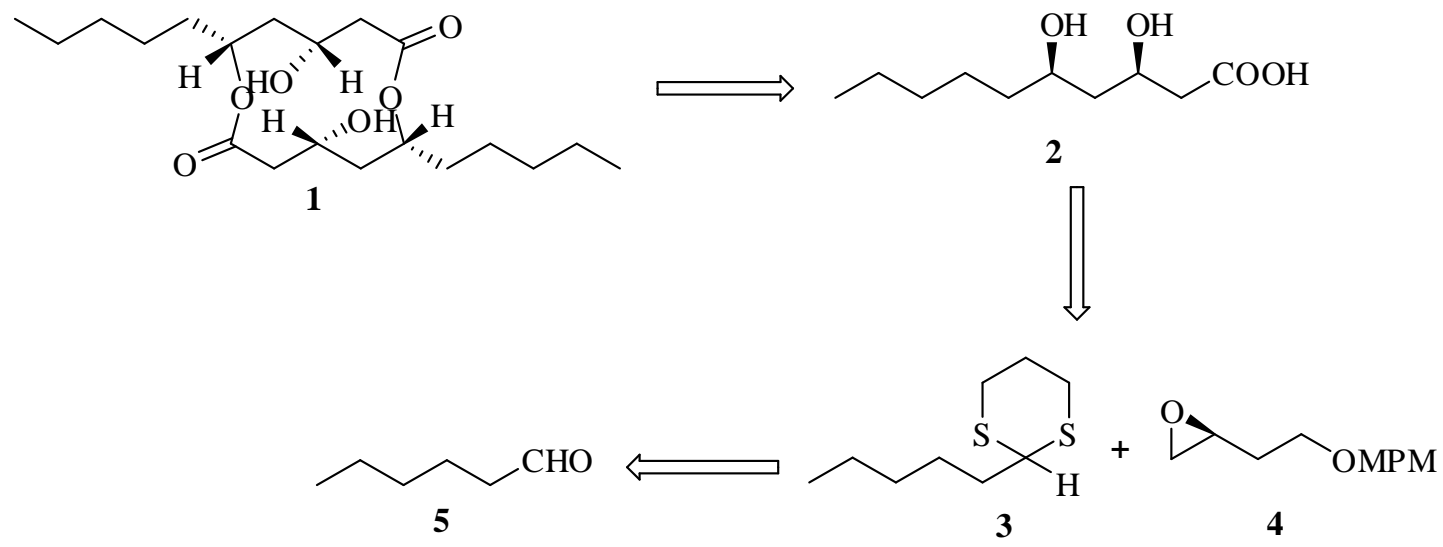

Scheme 1. Retrosynthetic route of Verbalactone.
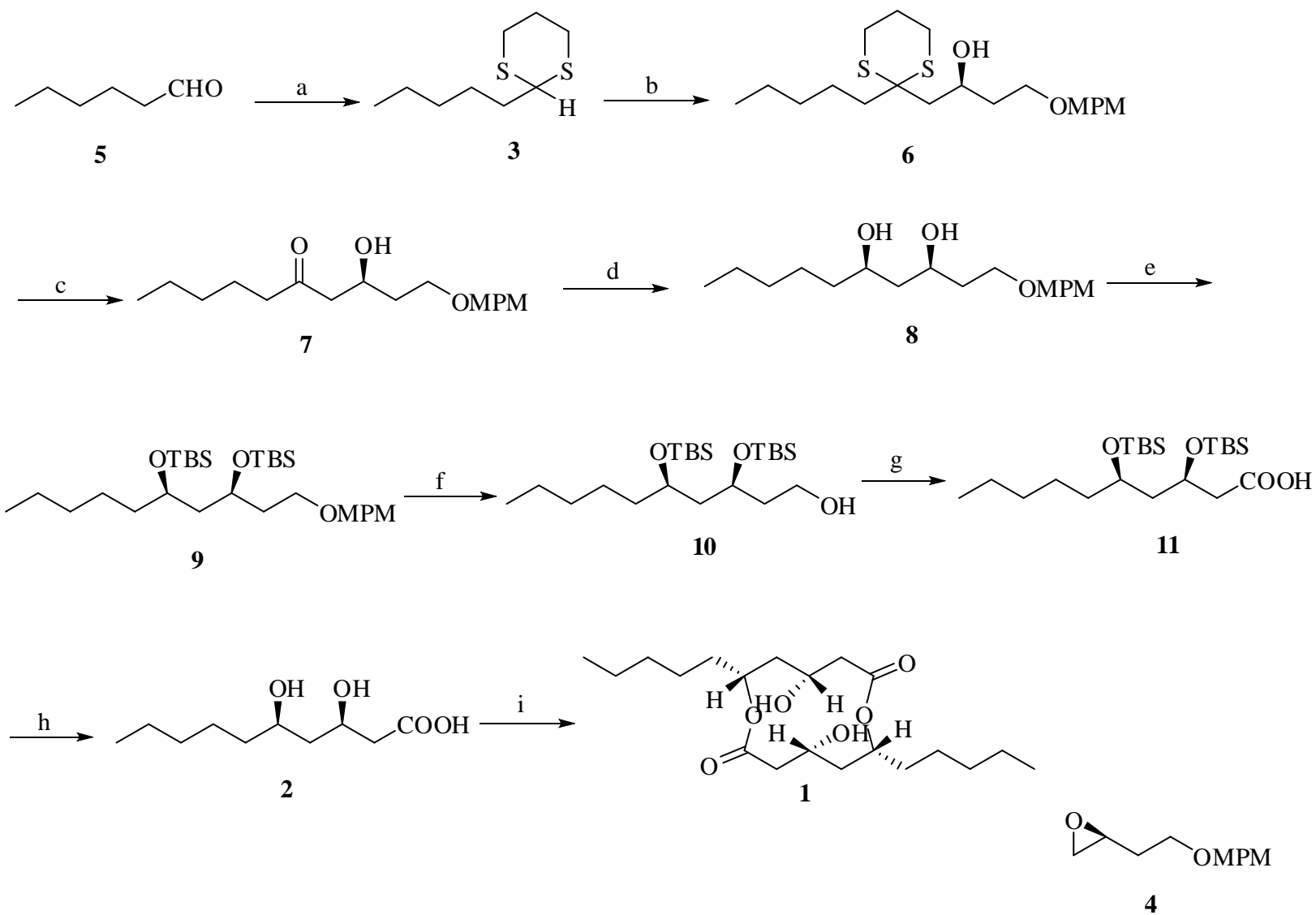

Reagents and conditions: (a) 1,3-propanedithiol, $\mathrm{BF}_{3} \cdot \mathrm{OEt}_{2}, \mathrm{CH}_{2} \mathrm{Cl}_{2}, 0{ }^{\circ} \mathrm{C}$-rt, $12 \mathrm{~h}$.(b) 4, $n$-BuLi, dry $\mathrm{THF},-78^{\circ} \mathrm{C}$ to $-20{ }^{\circ} \mathrm{C}$, 3h. (c) $\mathrm{CaCO}_{3}, \mathrm{I}_{2}, \mathrm{THF} / \mathrm{H}_{2} \mathrm{O}(4: 1), 0{ }^{\circ} \mathrm{C}, 30 \mathrm{~min}$ (d) catecholborane, dry $\mathrm{THF},-10{ }^{\circ} \mathrm{C}, 4 \mathrm{~h},(\mathrm{e}) \mathrm{TBSCl}$, Imidazole, $\mathrm{CH}_{2} \mathrm{Cl}_{2}$, rt, 4h; (f) DDQ, $\mathrm{CH}_{2} \mathrm{Cl}_{2}: \mathrm{H}_{2} \mathrm{O}$ (19:1), rt, 3 h. (g) TEMPO, BIAB, $\mathrm{H}_{2} \mathrm{O}: \mathrm{CH}_{2} \mathrm{Cl}_{2},(1: 1), 0{ }^{\circ} \mathrm{C}, 1 \mathrm{~h}$. (h) TBAF, THF, $0{ }^{\circ} \mathrm{C}$ to rt, $3 \mathrm{~h}$. (i) ) 2,4,6-trichlorobenzoyl chloride, $\mathrm{Et}_{3} \mathrm{~N}$, THF, rt, $2 \mathrm{~h}$ then DMAP, toluene, reflux, $6 \mathrm{~h}$

Scheme 2. Stereoselective total synthesis of Verbalactone.

The synthesis (Scheme 2) was started from the commercially available hexanal 5, which was converted as corresponding 1,3- dithiane using 1,3-propanedithiol and $\mathrm{BF}_{3} \cdot \mathrm{OEt}_{2}$ in $\mathrm{CH}_{2} \mathrm{Cl}_{2}$ for $12 \mathrm{~h}$. Later, 1,3- dithiane 3 on treatment with $n$-BuLi followed by the regioselective opening of known epoxide $4^{28}$ with resulting carbanion gave alcohol 6 in 77\% yield. Later, the 1,3-dithioacetal group was removed from 6 to furnish the $\beta$-hydroxy 
ketone 7. For this tranformation, we first tried with DDQ in aqueous MeCN. But in this reaction conditions the yield of the required compound is low $(<40 \%)$ and some of the Starting material remains unchanged. To overcome this, we next tried with $\mathrm{CaCO}_{3}, \mathrm{I}_{2}$, in $\mathrm{THF} / \mathrm{H}_{2} \mathrm{O}(4: 1)$, in this method the reaction took place with good yield (74\%). The stereoselective reduction of $\beta$-hydroxyl ketone $\mathbf{7}$ to the corresponding syn 1, 3-diol 8 was achieved using catecholborane (3.0 eq.) in THF at $-10{ }^{\circ} \mathrm{C}$ in $84 \%$ yield with high diastereoselectivity (syn: anti, 99:1). The resulting diol 8 was masked as silylethers with $\mathrm{TBSCl}$ and imidazole in $\mathrm{CH}_{2} \mathrm{Cl}_{2}$ to give 9 (81\%), which on selective cleavage of PMB ether in the presence of DDQ in aq. $\mathrm{CH}_{2} \mathrm{Cl}_{2}$ gave alcohol $\mathbf{1 0}$ in $78 \%$ yield. The alcohol 10 was oxidized to acid by treating with TEMPO and BIAB in aq. $\mathrm{CH}_{2} \mathrm{Cl}_{2}$ to afford acid $\mathbf{1 1}$ in $71 \%$ yield, which on desilylation with TBAF in dry THF afforded the dihydroxy acid $\mathbf{2}$.

Finally, The resulting dihydroxy acid $\mathbf{2}$ was immediately subjected to macrolactonisation under Yamaguchi reaction conditions (2,4,6-trichlorobenzoyl chloride, $\mathrm{Et}_{3} \mathrm{~N}$ and DMAP) to afford the target molecule Verbalactone 1 in in $57 \%$ yield. The ${ }^{1} \mathrm{H}$ NMR, ${ }^{13} \mathrm{C}$ NMR and optical rotation value of synthetic 1 were in good agreement with those of the previously reported natural product. ${ }^{21}$

\section{Conclusions}

In conclusion, a stereoselective total synthesis of Verbalactone $\mathbf{1}$ was accomplished by a versatile strategy. A combination of stereoselective reduction of $\beta$-hydroxyl ketone using catecholborane, regioselective ring opening of epoxide and Yamaguchi macrolactonisation were effectively utilized in accomplishing the synthesis.

\section{Experimental Section}

General. All chemicals and solvents were purchased from Sigma-Aldrich and Merck and used without further purification. All reactions were monitored by thin-layer chromatography (TLC) on silica Merck 60 F254 precoated aluminum plates. ${ }^{1} \mathrm{H}$ and ${ }^{13} \mathrm{C}$ NMR spectra were recorded with 500, 300, 150, and $75 \mathrm{MHz}$ Bruker spectrometer. Chemical shifts are reported in d units (ppm) with tetramethylsilane (TMS) as a reference. All coupling constants $(\mathrm{J})$ are reported in Hertz. Multiplicity is indicated as $\mathrm{s}$ (singlet), $\mathrm{d}$ (doublet), dd (double doublet), t (triplet), q (quartet), $m$ (multiplet). FT-IR spectra were taken on IR spectrophotometer using $\mathrm{NaCl}$ optics. Mass spectra were performed on direct inlet system or LC by MSD trap SL, the HRMS data were obtained using Q-TOF mass spectrometry. Optical rotation values are recorded on digital polarimeter at $25{ }^{\circ} \mathrm{C}$.

2-Pentyl-1,3-dithiane (3). ${ }^{29}$ To a stirred solution of hexanal $\left(5.0 \mathrm{~mL}, 49.07 \mathrm{mmol}\right.$ ) in $\mathrm{CH}_{2} \mathrm{Cl}_{2}$ (20 mL) was added 1,3-propanedithiol $(6.4 \mathrm{~mL}, 58.89 \mathrm{mmol})$. The mixture was cooled to $0{ }^{\circ} \mathrm{C}$ and $\mathrm{BF}_{3} . \mathrm{OEt}_{2}(3.0 \mathrm{~mL}, 24.53 \mathrm{mmol})$ was added dropwise. The mixture was allowed to warm to room temperature stirred for $12 \mathrm{~h}$. After completion of reaction the reaction was quenched by the addition of saturated aqueous $\mathrm{NaHCO}_{3}$. The mixture was diluted with $\mathrm{CH}_{2} \mathrm{Cl}_{2}$ and washed twice with $0.1 \mathrm{M} \mathrm{NaOH}$ then three times with brine. The combined aqueous phases were extracted twice with $\mathrm{CH}_{2} \mathrm{Cl}_{2}$ and the combined organic layers were dried over $\mathrm{NaSO}_{4}$ then concentrated and purified by Flash chromatography (silica gel, 60-120 mesh, 4\% EtOAc in pet. ether) afforded $3(8.0 \mathrm{~g} 86 \%)$ as a colorless oil. ${ }^{1} \mathrm{H} \mathrm{NMR}\left(300 \mathrm{MHz}, \mathrm{CDCl}_{3}\right): \delta 4.05(\mathrm{t}, \mathrm{J} 6.9 \mathrm{~Hz}, 1 \mathrm{H}), 2.91-2.80(\mathrm{~m}, 4 \mathrm{H})$, 2.15-2.09 (m, 1H), 1.90-1.82 (m, 1H), 1.76-1.72 (m, 2H), 1.51 (quintet, J 7.6 Hz, 2H), 1.33-1.25 (m, 4H), $0.89(\mathrm{t}, J$ $7.0 \mathrm{~Hz}, 3 \mathrm{H}) ;{ }^{13} \mathrm{C} \mathrm{NMR}\left(100 \mathrm{MHz}, \mathrm{CDCl}_{3}\right): \delta 47.9,35.6,31.6,30.7,26.5,26.3,22.6,14.2$; IR (neat) 2930, 1422, 1275, 1182, $908 \mathrm{~cm}^{-1}$; HRMS (ESI): $\mathrm{m} / z$ calcd for $\mathrm{C}_{9} \mathrm{H}_{18} \mathrm{NaS}_{2}: 213.0748$; found: 213.0751 [M+Na]. 
(S)-4-(4-Methoxybenzyloxy)-1-(2-pentyl-1,3-dithian-2-yl)butan-2-ol (6). To a solution of 3 (5.0 g, $26.31 \mathrm{mmol})$ in dry THF $(20 \mathrm{~mL})$ was added $n$-BuLi $\left(15.7 \mathrm{~mL}, 39.47 \mathrm{mmol}, 2.5 \mathrm{~N}\right.$ hexane solution) dropwise under $\mathrm{N}_{2}$ atmosphere at $-78{ }^{\circ} \mathrm{C}$ and stirred for $30 \mathrm{~min}$. then the reaction mixture was sequentially treated with a solution of epoxide $4(6.0 \mathrm{~mL}, 28.94 \mathrm{mmol})$ in dry THF $(15 \mathrm{~mL})$ at $10 \mathrm{~min}$ interval and stirred for an additional 3 h at $-20{ }^{\circ} \mathrm{C}$. After completion of reaction, Saturated $\mathrm{NaHCO}_{3}$ solution $(20 \mathrm{~mL})$ followed by saturated $\mathrm{NH}_{4} \mathrm{Cl}$ solution $(20 \mathrm{~mL})$ were added to the reaction mixture at $-20{ }^{\circ} \mathrm{C}$ and allowed to warm to room temperature. The reaction mixture was extracted with EtOAc $(2 \times 50 \mathrm{~mL})$. The organic layers were washed with water $(50 \mathrm{~mL})$, dried $\left(\mathrm{Na}_{2} \mathrm{SO}_{4}\right)$, evaporated and the residue obtained was purified by column chromatography (60-120 Silica

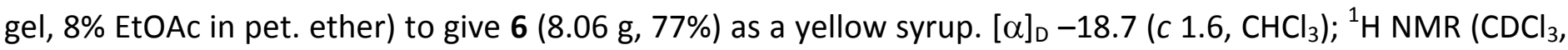
$300 \mathrm{MHz}$ ): $\delta 7.19(\mathrm{~d}, 2 \mathrm{H}, J 8.6 \mathrm{~Hz}, \mathrm{Ar}-\mathrm{H}), 6.83(\mathrm{~d}, 2 \mathrm{H}, J 8.6 \mathrm{~Hz}, \mathrm{Ar}-\mathrm{H}), 4.51\left(\mathrm{~d}, 1 \mathrm{H}, J 11.7 \mathrm{~Hz},-\mathrm{OCH}_{2} \mathrm{Ph}\right), 4.41(\mathrm{~d}$, $\left.1 \mathrm{H}, \mathrm{J} 11.7 \mathrm{~Hz},-\mathrm{OCH}_{2} \mathrm{Ph}\right), 3.81\left(\mathrm{~s}, 3 \mathrm{H},-\mathrm{OCH}_{3}\right), 3.75-3.79(\mathrm{~m}, 1 \mathrm{H}, \mathrm{H}-3), 3.48\left(\mathrm{t}, 2 \mathrm{H}, J 5.7 \mathrm{~Hz}, \mathrm{H}-1,1^{\prime}\right), 3.05(\mathrm{br} s, 1 \mathrm{H}$, $-\mathrm{OH}), 2.88-3.01(\mathrm{~m}, 2 \mathrm{H}), 2.66-2.75(\mathrm{~m}, 2 \mathrm{H}), 1.86-1.99(\mathrm{~m}, 2 \mathrm{H}), 1.55-1.79\left(\mathrm{~m}, 8 \mathrm{H}, 4 \times \mathrm{CH}_{2}\right), 1.26-1.34(\mathrm{~m}, 4 \mathrm{H}, 2 \times$ $\left.\mathrm{CH}_{2}\right), 0.92\left(\mathrm{t}, 3 \mathrm{H}, \mathrm{J} 5.7 \mathrm{~Hz},-\mathrm{CH}_{3}\right) ;{ }^{13} \mathrm{C} \mathrm{NMR}\left(75 \mathrm{MHz}, \mathrm{CDCl}_{3}\right): \delta 158.6,129.8,129.4,114.3,76.3,69.1,66.9,62.3$, 56.7, 44.8, 40.1, 38.3, 33.7, 27.3, 25.2, 23.3, 22.8, 14.6; IR (neat) 3373, 2984, 1372, 1175, $1082,928 \mathrm{~cm}^{-1}$; HRMS (ESI): $\mathrm{m} / \mathrm{z}$ calcd for $\mathrm{C}_{21} \mathrm{H}_{34} \mathrm{O}_{3} \mathrm{NaS}_{2}: 421.1847$; found: $421.1852[\mathrm{M}+\mathrm{Na}]^{+}$.

(S)-3-Hydroxy-1-(4-methoxybenzyloxy)decan-5-one (7). To a solution of compound 6 (4.1 g, $10.30 \mathrm{mmol}$ ) and $\mathrm{CaCO}_{3}$ $(8.24 \mathrm{~g}, 82.41 \mathrm{mmol})$ in $\mathrm{THF} / \mathrm{H}_{2} \mathrm{O}(\mathrm{v} / \mathrm{v}, 4: 1,100 \mathrm{~mL})$ was added iodide $(5.21 \mathrm{~g}, 20.6 \mathrm{mmol})$ at $0{ }^{\circ} \mathrm{C}$. The resulting mixture was stirred at $0{ }^{\circ} \mathrm{C}$ for $30 \mathrm{~min}$. The reaction was quenched by adding saturated aqueous $\mathrm{Na}_{2} \mathrm{~S}_{2} \mathrm{O}_{3}$, filtered through a pad of Celite, and then extracted with EtOAc $(3 \times 100 \mathrm{~mL})$, water, brine, dried over $\mathrm{Na}_{2} \mathrm{SO}_{4}$ and concentrated in vacuo. Purification by flash chromatography (silica gel, 60-120 mesh, 15\% EtOAc in pet. ether) to gave compound 7 (2.34 g, $74 \%$ yield) as colorless oil: $[\alpha]_{D}-98.7$ (c 1.6, $\left.\mathrm{CHCl}_{3}\right) ;{ }^{1} \mathrm{H} \mathrm{NMR}\left(\mathrm{CDCl}_{3}, 300 \mathrm{MHz}\right): \delta 7.22(\mathrm{~d}, 2 \mathrm{H}, J 8.8 \mathrm{~Hz}, \mathrm{Ar}-\mathrm{H}), 6.79(\mathrm{~d}, 2 \mathrm{H}, J$ $8.8 \mathrm{~Hz}, \mathrm{Ar}-\mathrm{H}$ ), 4.47 (d, 1H, J $\left.11.6 \mathrm{~Hz},-\mathrm{OCH}_{2} \mathrm{Ph}\right), 4.39\left(\mathrm{~d}, 1 \mathrm{H}, J 11.6 \mathrm{~Hz},-\mathrm{OCH}_{2} \mathrm{Ph}\right), 3.79\left(\mathrm{~s}, 3 \mathrm{H},-\mathrm{OCH}_{3}\right), 3.66-3.58(\mathrm{~m}, 1 \mathrm{H})$, $3.51\left(\mathrm{t}, 2 \mathrm{H}, J 6.1 \mathrm{~Hz}, 2.91(\mathrm{br} \mathrm{s}, 1 \mathrm{H},-\mathrm{OH}), 2.68-2.54(\mathrm{~m}, 2 \mathrm{H}), 2.06\left(\mathrm{t}, 2 \mathrm{H}, J 6.7 \mathrm{~Hz},-\mathrm{CH}_{2}\right), 1.52-1.39\left(\mathrm{~m}, 4 \mathrm{H}, 2 \times \mathrm{CH}_{2}\right), 1.29-\right.$ $1.18\left(\mathrm{~m}, 4 \mathrm{H}, 2 \times \mathrm{CH}_{2}\right), 0.89\left(\mathrm{t}, 3 \mathrm{H}, J 5.8 \mathrm{~Hz},-\mathrm{CH}_{3}\right) ;{ }^{13} \mathrm{C} \mathrm{NMR}\left(75 \mathrm{MHz}, \mathrm{CDCl}_{3}\right): \delta 206.7,159.0,129.7,129.4,114.1,76.6,68.3$, 67.9, 56.6, 50.1, 40.9, 36.6, 32.9, 26.7, 14.1; HRMS (ESI): $\mathrm{m} / z$ calcd for $\mathrm{C}_{18} \mathrm{H}_{28} \mathrm{O}_{4} \mathrm{Na}$ : 331.1885; found: 331.1890 [M+Na] $]^{+}$

(3S,5R)-1-(4-Methoxybenzyloxy)decane-3,5-diol (8). To a stirred solution of compound 7 (2.25 g, $7.30 \mathrm{mmol})$ in dry tetrahydrofuran, freshly distilled catecholborane $\left(2.3 \mathrm{~mL}, 21.9 \mathrm{mmol}\right.$ ) was added at $-10{ }^{\circ} \mathrm{C}$ (reaction mixture was kept in $\mathrm{MeOH}$-ice bath). After $4 \mathrm{~h}$, the reaction mixture was quenched by the addition of $1 \mathrm{~mL}$ of anhydrous $\mathrm{MeOH}$ and $2 \mathrm{~mL}$ of a saturated aqueous solution of sodium potassium tartarate. This mixture was allowed to stir at room temperature for $2 \mathrm{~h}$. The layers were separated, and aqueous layer was extracted with ethyl acetate $(3 \times 30 \mathrm{ml})$. The organic extracts were washed with brine $(30 \mathrm{ml})$ and dried over anhydrous $\mathrm{Na}_{2} \mathrm{SO}_{4}$, and purified by column chromatography (silica gel, 60-120 mesh, 25\% EtOAc in pet. ether) to afford the diol 8 (1.9 g, 84\%) as a liquid. $[\alpha]_{\mathrm{D}}-38.1$ (c 1.6, $\left.\mathrm{CHCl}_{3}\right) ;{ }^{1} \mathrm{H} \mathrm{NMR}\left(\mathrm{CDCl}_{3}, 300 \mathrm{MHz}\right): \delta 7.22(\mathrm{~d}, 2 \mathrm{H}, J 8.8 \mathrm{~Hz}$, $\mathrm{Ar}-\mathrm{H}), 6.79(\mathrm{~d}, 2 \mathrm{H}, \mathrm{J} 8.8 \mathrm{~Hz}, \mathrm{Ar}-\mathrm{H}), 4.47\left(\mathrm{~d}, 1 \mathrm{H}, J 11.6 \mathrm{~Hz},-\mathrm{OCH}_{2} \mathrm{Ph}\right), 4.39\left(\mathrm{~d}, 1 \mathrm{H}, J 11.6 \mathrm{~Hz},-\mathrm{OCH}_{2} \mathrm{Ph}\right), 3.83(\mathrm{~s}, 3 \mathrm{H}$, $\left.-\mathrm{OCH}_{3}\right), 3.76-3.64(\mathrm{~m}, 2 \mathrm{H}), 3.43(\mathrm{t}, 2 \mathrm{H}, \mathrm{J} 5.8 \mathrm{~Hz}), 3.03-2.88(\mathrm{br} \mathrm{s}, 2 \mathrm{H}, 2 \mathrm{x}-\mathrm{OH}), 1.78-1.64\left(\mathrm{~m}, 4 \mathrm{H}, 2 \mathrm{x}-\mathrm{CH}_{2}\right), 1.52-$ $1.28\left(\mathrm{~m}, 8 \mathrm{H}, 4 \times \mathrm{CH}_{2}\right), 0.83\left(\mathrm{t}, 3 \mathrm{H}, J 5.8 \mathrm{~Hz},-\mathrm{CH}_{3}\right) ;{ }^{13} \mathrm{C} \mathrm{NMR}\left(75 \mathrm{MHz}, \mathrm{CDCl}_{3}\right): \delta 158.7,129.7,129.5,114.3,76.7$, $67.9,67.5,67.3,56.1,45.6,36.3,33.8,32.7,25.6,14.2$; HRMS (ESI): $\mathrm{m} / z$ calcd for $\mathrm{C}_{18} \mathrm{H}_{30} \mathrm{O}_{4} \mathrm{Na}: 333.2042$; found: $333.2047[\mathrm{M}+\mathrm{Na}]^{+}$.

(5S,7R)-5-(2-(4-Methoxybenzyloxy)ethyl)-2,2,3,3,9,9,10,10-octamethyl-7-pentyl-4,8-dioxa-3,9-disilaun-

decane (9). A mixture of the above alcohol $8(1.74 \mathrm{~g}, 5.61 \mathrm{mmol})$ and imidazole $(1.52 \mathrm{~g}, 22.45 \mathrm{mmol})$ in dry $\mathrm{CH}_{2} \mathrm{Cl}_{2}(40 \mathrm{~mL})$ was treated with $\mathrm{TBSCl}(2.10 \mathrm{~g}, 14.02 \mathrm{mmol})$ at $0{ }^{\circ} \mathrm{C}$ under nitrogen atmosphere and stirred at room temperature for $4 \mathrm{~h}$. The reaction mixture was quenched with aq. $\mathrm{NH}_{4} \mathrm{Cl}$ solution $(10 \mathrm{~mL})$ and extracted with $\mathrm{CH}_{2} \mathrm{Cl}_{2}(2 \times 50 \mathrm{~mL})$. The combined extracts were washed with water $(30 \mathrm{~mL})$, brine $(30 \mathrm{~mL})$, dried $\left(\mathrm{Na}_{2} \mathrm{SO}_{4}\right)$ and concentrated. The residue was purified by column chromatography (60-120 Silica gel, 5\% EtOAc in pet. 
ether) to furnish $9(2.44 \mathrm{~g}, 81 \%)$ as a colorless liquid. $[\alpha]_{\mathrm{D}}-57.4\left(c \mathrm{0.76}, \mathrm{CHCl}_{3}\right) ;{ }^{1} \mathrm{H} \mathrm{NMR}\left(\mathrm{CDCl}_{3}, 300 \mathrm{MHz}\right): \delta$ 7.22 (d, 2H, J $8.8 \mathrm{~Hz}, \mathrm{Ar}-\mathrm{H}), 6.79$ (d, $2 \mathrm{H}, J 8.8 \mathrm{~Hz}, \mathrm{Ar}-\mathrm{H}), 4.47$ (d, $\left.1 \mathrm{H}, J 11.6 \mathrm{~Hz},-\mathrm{OCH}_{2} \mathrm{Ph}\right), 4.39(\mathrm{~d}, 1 \mathrm{H}, J 11.6 \mathrm{~Hz},-$ $\left.\mathrm{OCH}_{2} \mathrm{Ph}\right), 3.83\left(\mathrm{~s}, 3 \mathrm{H},-\mathrm{OCH}_{3}\right), 3.52-3.41(\mathrm{~m}, 2 \mathrm{H}), 3.41(\mathrm{t}, 2 \mathrm{H}, \mathrm{J} 6.0 \mathrm{~Hz}), 1.74-1.56\left(\mathrm{~m}, 6 \mathrm{H}, 3 \times \mathrm{CH}_{2}\right), 1.42-1.25(\mathrm{~m}$, $\left.6 \mathrm{H}, 3 \mathrm{x}-\mathrm{CH}_{2}\right), 0.88\left(\mathrm{t}, 3 \mathrm{H}, \mathrm{J} 5.8 \mathrm{~Hz},-\mathrm{CH}_{3}\right), 0.83\left(\mathrm{~s}, 9 \mathrm{H}, \mathrm{t}-\right.$ butyl), $0.79\left(\mathrm{~s}, 9 \mathrm{H}, \mathrm{t}-\right.$ butyl) $, 0.08\left(\mathrm{~s}, 6 \mathrm{H}, 2 \mathrm{x}-\mathrm{CH}_{3}\right), 0.03(\mathrm{~s}$, $\left.6 \mathrm{H}, 2 \mathrm{x}-\mathrm{CH}_{3}\right) ;{ }^{13} \mathrm{C} \mathrm{NMR}\left(75 \mathrm{MHz}, \mathrm{CDCl}_{3}\right): \delta 158.6,129.7,129.4,114.2,113.2,75.9,70.3,67.9,67.6,56.0,46.1$, 36.2, 33.7, 31.9, 26.6, 26.3, 25.7, 22.8, 17.9, 13.9, -4.3, -4.6; HRMS (ESI): $\mathrm{m} / \mathrm{z}$ calcd for $\mathrm{C}_{30} \mathrm{H}_{58} \mathrm{O}_{4} \mathrm{NaSi}_{2}$ : 561.3771; found: $561.3774[\mathrm{M}+\mathrm{Na}]^{+}$.

(3S,5R)-3,5-Bis(tert-butyldimethylsilyloxy)decan-1-ol (10). To a solution of 9 (2.3 g, $4.27 \mathrm{mmol}$ ) in aq. $\mathrm{CH}_{2} \mathrm{Cl}_{2}$ $(20 \mathrm{~mL}, 19: 1)$, DDQ (1.16 g, $5.13 \mathrm{mmol})$ was added and stirred at room temperature for $3 \mathrm{~h}$. The reaction mixture was quenched with sat. $\mathrm{NaHCO}_{3}$ solution $(30 \mathrm{~mL})$, filtered and washed with $\mathrm{CH}_{2} \mathrm{Cl}_{2}(50 \mathrm{~mL})$. The filtrate was washed with water $(30 \mathrm{~mL})$, brine $(30 \mathrm{~mL})$, dried $\left(\mathrm{Na}_{2} \mathrm{SO}_{4}\right)$ and evaporated under reduced pressure. The residue was purified by column chromatography (60-120 Silica gel, 15\% EtOAc in pet. ether) to furnish 10 (1.39 g, 78\%). $[\alpha]_{D}-57.4\left(c 0.76, \mathrm{CHCl}_{3}\right) ;{ }^{1} \mathrm{H}$ NMR $\left(\mathrm{CDCl}_{3}, 300 \mathrm{MHz}\right): \delta 3.72-3.61(\mathrm{~m}, 2 \mathrm{H}), 3.57(\mathrm{br} \mathrm{s}, 1 \mathrm{H},-\mathrm{OH}), 3.47$ $(\mathrm{m}, 1 \mathrm{H}), 3.41(\mathrm{~m}, 1 \mathrm{H}), 1.82-1.66\left(\mathrm{~m}, 8 \mathrm{H}, 4 \times \mathrm{CH}_{2}\right), 1.39-1.21\left(\mathrm{~m}, 2 \mathrm{H},-\mathrm{CH}_{2}\right), 1.19-1.11\left(\mathrm{~m}, 2 \mathrm{H},-\mathrm{CH}_{2}\right), 0.87(\mathrm{t}, 3 \mathrm{H}, \mathrm{J}$ $\left.5.8 \mathrm{~Hz},-\mathrm{CH}_{3}\right), 0.81\left(\mathrm{~s}, 9 \mathrm{H}, \mathrm{t}\right.$-butyl), $0.79\left(\mathrm{~s}, 9 \mathrm{H}, \mathrm{t}\right.$-butyl), $0.07\left(\mathrm{~s}, 6 \mathrm{H}, 2 \mathrm{x}-\mathrm{CH}_{3}\right), 0.01\left(\mathrm{~s}, 6 \mathrm{H}, 2 \mathrm{x}-\mathrm{CH}_{3}\right) ;{ }^{13} \mathrm{C} \mathrm{NMR}(75$ $\mathrm{MHz}_{\mathrm{CDCl}}$ ): $\delta$ 70.3, 69.0, 61.3, 46.3, 38.3, 35.2, 32.3, 26.3, 26.1, 25.3, 23.1, 19.1, 14.0, -4.4, -4.7; HRMS (ESI): $\mathrm{m} / \mathrm{z}$ calcd for $\mathrm{C}_{22} \mathrm{H}_{50} \mathrm{O}_{3} \mathrm{NaSi}_{2}: 441.3196$; found: $441.3199[\mathrm{M}+\mathrm{Na}]^{+}$.

(3R,5R)-3,5-Bis(tert-butyldimethylsilyloxy)decanoic acid (11). To a stirred solution of 10 (1.20 g, $2.87 \mathrm{mmol})$ in $\mathrm{CH}_{2} \mathrm{Cl}_{2}: \mathrm{H}_{2} \mathrm{O}(1: 1,10 \mathrm{~mL})$, TEMPO $(0.15 \mathrm{~g}, 0.97 \mathrm{mmol})$ and $\mathrm{BIAB}(2.77 \mathrm{~g}, 8.61 \mathrm{mmol})$ were added at $0{ }^{\circ} \mathrm{C}$ and stirred for $1 \mathrm{~h}$. The reaction mixture was diluted with water $(5 \mathrm{~mL})$ and extracted with $\mathrm{CH}_{2} \mathrm{Cl}_{2}(2 \times 20 \mathrm{~mL})$. The organic layers were washed with brine $(10 \mathrm{~mL})$, dried $\left(\mathrm{Na}_{2} \mathrm{SO}_{4}\right)$, evaporated and the residue purified by column chromatography (silica gel, 60-120 mesh, 20\% EtOAc in pet. ether) to give acid 11 (0.88 g, 71\%) as a colorless gummy oil. $[\alpha]_{D}-7.4\left(c 0.9, \mathrm{CHCl}_{3}\right) .{ }^{1} \mathrm{H} \mathrm{NMR}\left(\mathrm{CDCl}_{3}, 300 \mathrm{MHz}\right): \delta 4.26-4.22(\mathrm{~m}, 1 \mathrm{H}) ; 3.73-3.67(\mathrm{~m}, 1 \mathrm{H}) ; 2.59$ (dd, J 15.0, 5.0 Hz, $1 \mathrm{H}$ ); 2.45 (dd, J 15.0, $6.8 \mathrm{~Hz}, 1 \mathrm{H}) ; 1.78-1.55(\mathrm{~m}, 2 \mathrm{H}) ; 1.45-1.12(\mathrm{~m}, 8 \mathrm{H}) ; 0.91(\mathrm{t}, 3 \mathrm{H}, J 7.1$ $\left.\mathrm{Hz},-\mathrm{CH}_{3}\right), 0.81$ (s, 9H, t-butyl), $0.79\left(\mathrm{~s}, 9 \mathrm{H}, t\right.$-butyl); $0.07(\mathrm{~s}, 6 \mathrm{H}) ; 0.04(\mathrm{~s}, 6 \mathrm{H}) .{ }^{13} \mathrm{C} \mathrm{NMR}\left(75 \mathrm{MHz}, \mathrm{CDCl}_{3}\right): \delta 176.9$, 69.3, 66.8, 44.4, 42.1, 37.3, 32.1, 29.8, 25.8, 25.7, 24.6, 22.6, 17.9, 17.8, 14.0, -4.2, -4.5, -4.8; HRMS (ESI): $\mathrm{m} / z$ calcd for $\mathrm{C}_{22} \mathrm{H}_{48} \mathrm{O}_{4} \mathrm{NaSi}_{2}: 455.2989$; found: $455.2994[\mathrm{M}+\mathrm{Na}]^{+}$.

(3R,5R)-3,5-Dihydroxydecanoic acid (2). To a cooled $\left(0^{\circ} \mathrm{C}\right)$ solution of 11 (0.72 g, $\left.1.66 \mathrm{mmol}\right)$ in dry THF (5 mL) under nitrogen atmosphere, TBAF $(4.2 \mathrm{~mL}, 4.2 \mathrm{mmol})$ was added and stirred for $3 \mathrm{~h}$. After completion of reaction, reaction mixture was diluted with water $(5 \mathrm{~mL})$ and extracted with ethyl acetate $(2 \times 20 \mathrm{~mL})$. Organic layers were washed with water $(2 \times 10 \mathrm{~mL})$, brine $(10 \mathrm{~mL})$, dried $\left(\mathrm{Na}_{2} \mathrm{SO}_{4}\right)$, evaporated and purified the residue by colomn chromatography to give $2(0.26 \mathrm{~g}, 78 \%)$ as semisolid which was directly used for next reaction without any purification.

Verbalactone (1). To a stirred solution of $2(0.26 \mathrm{~g}, 1.27 \mathrm{mmol})$ and $\mathrm{Et}_{3} \mathrm{~N}(0.53 \mathrm{~mL}, 3.81 \mathrm{mmol})$ in dry THF (2 $\mathrm{mL})$, a solution of 2, 4, 6-trichlorobenzoyl chloride $(0.30 \mathrm{~mL}, 1.91 \mathrm{mmol})$ in dry THF $(1 \mathrm{~mL})$ was added. The resulting mixture was stirred for $2 \mathrm{~h}$ at room temperature under nitrogen atmosphere and evaporated to afford the mixed anhydride. It was diluted with toluene $(10 \mathrm{~mL})$ and filtered quickly through celite. The filtrate was added drop wise to a stirred solution of DMAP $(1.54 \mathrm{~g}, 12.7 \mathrm{mmol})$ in toluene $(35 \mathrm{~mL})$ at $90{ }^{\circ} \mathrm{C}$ over a period of $6 \mathrm{~h}$. After the complete addition, the reaction mixture was stirred at $100{ }^{\circ} \mathrm{C}$ for $2 \mathrm{~h}$. It was cooled, washed with $7 \%$ aq $\mathrm{NaHCO}_{3}(40 \mathrm{~mL}), 2 \mathrm{M}$ aqueous $\mathrm{HCl}(40 \mathrm{~mL})$, brine $(40 \mathrm{~mL})$ and dried $\left(\mathrm{Na}_{2} \mathrm{SO}_{4}\right)$. The organic layer was evaporated and the obtained residue purified by column chromatography (60-120 Silica gel, $15 \%$ EtOAc in pet. ether) to give $1(0.13 \mathrm{~g}, 57 \%)$ as a syrup. $[\alpha]_{\mathrm{D}}+10.1$ (c $\left.1.1, \mathrm{CHCl}_{3}\right) .{ }^{1} \mathrm{H}-\mathrm{NMR}\left(\mathrm{CDCl}_{3}, 300 \mathrm{MHz}\right): \delta$ 4.97-4.90 (m, $2 \mathrm{H}, 2$ x -CH); 4.11-4.04 (m, 2 H, 2 x-CH); 3.73 (br s, 2 x OH); 2.71 (d, 4 H, J 3.1 Hz, 2 x -CH2); 2.04 (ddd, $2 \mathrm{H}, J$ 15.3, 10.2, 3.1 Hz, 2 x -CH); 1.98 (td, $2 \mathrm{H}, J$ 4.0, $15.0 \mathrm{~Hz}, 2 \mathrm{x}-\mathrm{CH}) ; 1.69-1.39$ (m, $4 \mathrm{H}, 2 \mathrm{x}-\mathrm{CH} 2) ; 1.37-$ 
$\left.1.20(\mathrm{~m}, 12 \mathrm{H}, 6 \times \mathrm{CH} 2) ; 0.85(\mathrm{t}, 6 \mathrm{H}, J 6.3 \mathrm{~Hz}, 2 \times-\mathrm{CH} 3) .{ }^{13} \mathrm{CNMR}_{(\mathrm{CDCl}}, 75 \mathrm{MHz}\right): \delta 173.1,72.7,64.9,39.3$, 38.1, 31.6, 31.5, 25.4, 22.5, 14.3; HRMS (ESI): $\mathrm{m} / z$ calcd for $\mathrm{C}_{20} \mathrm{H}_{36} \mathrm{O}_{6} \mathrm{Na:} 395.2410$; found: 395.2414 [M+Na] ${ }^{+}$.

\section{Acknowledgements}

The autohrs are thankful to Jawaharlal Nehru Technological University Hyderabad, CMR Institute of Technology Secunderabad, QIS College of Engineering \& Technology (Autonomous), Vengamukkapalem, Ongole for constant encouragement in providing laboratory facilities and analytical data.

\section{Supplementary Material}

Copies of ${ }^{1} \mathrm{H}$ and ${ }^{13} \mathrm{C}$ NMR spectra associated with this paper can be found in the online version.

\section{References}

1. Edukondalu, P.; Sreenivasulu, R.; Chiranjeevi, B.; Kumar, V. N.; Raju, R. R. Monatsh. Chem. 2015, $146,1309$. https://doi.org/10.1007/s00706-014-1406-3

2. Pratapareddy, B.; Sreenivasulu, R.; Thota, P.; Hatti, I.; Rao, M. V. B.; Kumar, V.N.; Raju, R. R. Monatsh. Chem. 2016, 148, 751. https://doi.org/10.1007/s00706-016-1754-2

3. Ramakrishna, K.; Sreenivasulu, R.; Vidavalur, S.; Reddy, B. J. M. Lett. Org. Chem. 2016, 13, 693. https://doi.org/10.2174/1570178613666161013145238

4. Pratapareddy, B.; Sreenivasulu, R.; Hatti, I.; Rao, M. V. B.; Raju, R. R. Monatsh. Chem., 2015, $146,1921$. https://doi.org/10.1007/s00706-015-1464-1

5. Alluraiah, G.; Sreenivasulu, R.; Sadanandam, P.; Anitha, K.; Raju, R. R. Monatsh. Chem. 2016, $147,451$. https://doi.org/10.1007/s00706-015-1526-4

6. Murthy, I.S.; Sreenivasulu, R.; Alluraiah, G.; Raju, R. R. Lett. Org. Chem. 2014, 11, 327. https://doi.org/10.2174/1570178611666140124001937

7. Kind, R.; Zeeck, A.; Grabley, S.; Thiericke, R.; Zerlin, M. J. Nat. Prod. 1996, 59, 539. https://doi.org/10.1021/np960083q

8. Ghisalberti, E.L.; Hargreaves, J. R.; Skelton, B. W.; White, A. H. Aust. J. Chem. 2002, 55, 233. https://doi.org/10.1071/CH01197

9. Kis, Z.; Furger, P.; Sigg, H. Experientia 1969, 25, 123. https://doi.org/10.1007/BF01899073

10. Krohn, K.; Farooq, U.; Florke, U.; Schulz, B.; Draeger, S.; Pescitelli, G.; Salvadori, P.; Antus, S.; Kurtan, T. Eur. J. Org. Chem. 2007, 3206. https://doi.org/10.1002/ejoc.200601128

11. Kastanias, M. A.; Chrysayi-Tokousbalides, M. Pest. Manage. Sci. 2000, 56, 227. https://doi.org/10.1002/(SICI)1526-4998(200003)56:3<227::AID-PS115>3.0.CO;2-A

12. Magiatis, P.; Spanakis, D.; Mitaku, S.; Tsitsa, E.; Mentis, A.; Harvala, C. J. Nat. Prod. 2001, 64, 1093. https://doi.org/10.1021/np010025r

13. Takano, S.; Seton, M.; Ogasawara, K. Tetrahedron: Asymmetry 1992, 3, 533. 
https://doi.org/10.1016/S0957-4166(00)80259-1

14. Bennett, F.; Knight, D.; Fenton, G. J. Chem. Soc., Perkin Trans. 1 1991, 1543. https://doi.org/10.1039/P19910001543

15. Bennett, F.; Knight, D.; Fenton, G. Heterocycles 1989, 29, 639.

https://doi.org/10.3987/COM-89-4853

16. Yang, Y.L.; Falck, J.R. Mevinic acids and analogues: preparation of a key chiral intermediate. Tetrahedron Lett. 1982, 23, 4305.

https://doi.org/10.1016/S0040-4039(00)85585-9

17. Gogoi, S.; Barua, N. C.; Kalita, B. First total synthesis of verbalactone, a macrocyclic dilactone isolated from Verbascum undulatum. Tetrahedron Lett. 2004, 45, 5577.

https://doi.org/10.1016/i.tetlet.2004.05.149

18. Sharma, G. V. M.; Reddy, Ch. G. A stereoselective synthesis of verbalactone - determination of absolute stereochemistry. Tetrahedron Lett. 2004, 45, 7483.

https://doi.org/10.1016/i.tetlet.2004.08.052

19. Allais, F.; Louvel, M. C.; Cossy, J. Synlett 2007, 18, 451.

https://doi.org/10.1055/s-2007-967946

20. Salunke, G.B.; Shivakumar, I.; Gurjar, M. K. Tetrahedron Lett. 2009, 50, 2048.

https://doi.org/10.1016/j.tetlet.2009.02.062

21. Garg, A.; Singh, V.K. Tetrahedron 2009, 65, 8677.

https://doi.org/10.1016/j.tet.2009.08.054

22. Das, B.; Laxmminarayana, K.; Krishnaiah, M.; Nandan Kumar, D. Helv. Chim. Acta 2009, 92, 1840. https://doi.org/10.1002/hlca.200900083

23. Sabitha, G.; Bhaskar, V.; Yadav, J. S. Synth. Commun. 2008, 38, 3129.

https://doi.org/10.1080/00397910802055294

24. Wu, J.Z.; Gao, J.; Ren, G. B.; Zhen, Z. B.; Zhang, Y.; Wu, Y. Tetrahedron 2009, 65, 289. https://doi.org/10.1016/i.tet.2008.10.050

25. Alluraiah, G.; Sreenivasulu, R.; Murthy, I. S.; Raju, R. R. Monatsh. Chem. 2014, 145, 2019. https://doi.org/10.1007/s00706-014-1272-z

26. Madala, M.; Raman, B.; Sastry, K. V.; Musulla, S. Monatsh. Chem. 2016, 147, 1985. https://doi.org/10.1007/s00706-016-1682-1

27. Mineeva, I. V. Russ. J. Org. Chem. 2012, 48, 977. https://doi.org/10.1134/S1070428012070159

28. Nookaraju, U.; Begari, E. Organic and Biomolecular Chemistry, 2014, 12, 5973. https://doi.org/10.1039/C4OB00965G

29. Huckins, J. R.; Rychnovsky, S. D. J. Org. Chem. 2003, 68, 10135. https://doi.org/10.1021/jo035260o 